\title{
4 Forschungsdesign und methodisches Vorgehen
}

Das Forschungsdesign meiner Studie leitet sich aus dem Ziel ab, empirisch begründetes Wissen zum Zusammenhang des Handelns traditioneller Autoritäten und Konflikten um Land im Kontext von land grabbing zu schaffen. Dazu analysiere ich zwei Konflikte um die großflächige agrarindustrielle Umnutzung von Land in der Ashanti Region und Northern Region Ghanas. In beiden Fällen sind traditionelle Autoritäten als zentrale Akteure in die Konflikte involviert. Auf welche Weise traditionelle Autoritäten handeln und welche Rolle sie in Konflikten, die im Zusammenhang mit land grabbing auftreten, einnehmen, ist immer kontextabhängig und damit unter Berücksichtigung der jeweiligen Bedingungen zu analysieren. Dennoch erlauben die Erkenntnisse meiner Forschung es, Schlussfolgerungen zu ziehen, deren Reichweite über die untersuchten Fälle und den Länderkontext hinausgeht. Dazu tragen das vergleichende Forschungsdesign sowie Parallelen in der traditionellen Herrschaft unterschiedlicher postkolonialer Staaten bei, die auf ihre gemeinsame Kolonialvergangenheit zurückzuführen sind.

Im Folgenden stelle ich zunächst das Forschungsdesign meiner Studie vor, lege die Kriterien der Fallauswahl dar und gehe auf den Vergleich der beiden Fälle ein. Daran anschließend erläutere ich die für die Datenerhebung angewandten Methoden sowie meine Vorgehensweise bei der Datenauswertung.

\subsection{Kollektive Fallstudie}

Meine Forschung ist als eine Fallstudie mit instrumenteller Ausrichtung konzipiert (Stake 1995; Stake 2003). Eine Fallstudie ist die ausführliche empirische Untersuchung eines Phänomens unter Berücksichtigung des jeweiligen lebensweltlichen Kontexts (Yin 2009: 18). Ein Fall ist eine räumlich begrenz- 
te Einheit, die zu einem bestimmten Zeitpunkt oder über einen begrenzten Zeitraum hinweg erforscht wird (Gerring 2007: 19). In einer instrumentellen Fallstudie dient der Fall dazu, ein allgemeines Verständnis für ein spezifisches Phänomen zu entwickeln. Instrumentelle Fallstudien eignen sich insbesondere, um Hypothesen hinsichtlich eines bisher wenig erforschten Phänomens $\mathrm{zu}$ entwickeln. Je nach Erkenntnisinteresse kann es - wie auch für meine Studie - sinnvoll sein, mehr als nur einen einzigen Fall zu erforschen. Die Ausweitung instrumenteller Fallstudien auf mehrere Fälle bezeichnet Robert Stake (2003: 138) als kollektive Fallstudie.

Ein wichtiger Ausgangspunkt für die Analyse des Handelns traditioneller Autoritäten in Konflikten um Land ist die unterschiedliche Ausgestaltung traditioneller Herrschaft. Daher vergleiche ich zwei Fälle, in denen sich das traditionelle Herrschaftssystem unterscheidet. Ein Paarvergleich, (paired comparison) (Tarrow 2010) - wie ich ihn in meiner Studie durchführe - bietet im Verhältnis zu einer Einzelfallstudie eine ausgewogene Kombination aus deskriptiver Tiefe und analytischer Herausforderung. Der Schritt von einem Einzelfall hin $\mathrm{zu}$ einem Paarvergleich erlaubt es, verallgemeinerbare Hypothesen $\mathrm{zu}$ generieren und gleichzeitig in der Lage zu sein, zu überprüfen wie unterschiedliche Variablen interagieren.

\subsection{Kriterien der Fallauswahl}

In der Logik meiner Studie ist ein Fall ein Konflikt um Land im Kontext von land grabbing, in dem traditionelle Autoritäten zu den zentralen Konfliktakteuren zählen. Land grabbing verstehe ich in Anlehnung an Borras et al. (2012: 405) als die Aneignung von Kontrolle über relativ große Flächen Land und andere Ressourcen, die mit einer intendierten Inwertsetzung einhergeht und eine Verlagerung weg von der Ressourcennutzung hin zur Ressourcengewinnung für den heimischen oder globalen Markt mit sich bringt. Um den Fall zeitlich und räumlich einzugrenzen, eignen sich empirisch beobachtbare Konflikte, die im Zusammenhang mit der großflächigen Umnutzung von Land im Rahmen agrarindustrieller Projekte auftreten. Empirisch beobachtbare Konflikte verstehe ich als soziale Beziehungen zwischen mindestens zwei individuellen oder kollektiven Akteuren, gekennzeichnet durch von ihnen als widersprüchlich wahrgenommene Interessen, Ziele oder Bedürfnisse (Bonacker/Imbusch 2006), die in Handeln resultieren (Dietz/Engels 2014). 
Für die Fallauswahl setzen diese Kriterien voraus, dass es sich um einen Länderkontext handelt, in dem land grabbing relevant ist und traditionelle Autoritäten im Vergleich zu staatlichen Institutionen eine starke Stellung in der Verwaltung und Vergabe von Land sowie in der Regelung von Konflikten einnehmen. Aufgrund der theoretischen Vorannahme, dass die unterschiedliche Ausgestaltung traditioneller Herrschaft relevant für das Handeln traditioneller Autoritäten in Konflikten sein könnte, beziehe ich mich in meinem Vergleichsdesign auf die diverse-case-Strategie nach John Gerring (2007: 97). In diesem Fallstudiendesign zeichnen sich die einzelnen Fälle dadurch aus, dass sie sich hinsichtlich eines Aspekts oder einer Variable unterscheiden, während sie ansonsten eine gute Vergleichbarkeit charakterisiert. Eine Varianz bezüglich zentraler Aspekte des theoriegeleiteten Erkenntnisinteresses erhöht die Möglichkeit, Erkenntnisse und Schlussfolgerungen auf andere Kontexte übertragen zu können (Gerring 2007: 97f.). Daraus ergibt sich ein weiteres Kriterium für die Fallauswahl: die unterschiedliche Ausgestaltung der traditionellen Herrschaft. Für eine gute Vergleichbarkeit sollten sich andere intervenierende Kontextfaktoren durch eine größtmögliche Ähnlichkeit auszeichnen. Der Vergleich innerhalb desselben Länderkontexts ermöglicht es, die nationalen politischen und ökonomischen Kontextfaktoren stabil zu halten, die in der Literatur als relevant in Bezug auf Konflikte um Land im Kontext von land grabbing gelten (Hall et al. 2015). Darüber hinaus habe ich bei der Fallauswahl auf eine gute Vergleichbarkeit hinsichtlich des Zeitpunkts der Landvergabe, des Ausmaßes der gepachteten Fläche, des Zwecks der Umnutzung des Landes und der vorherigen Landnutzung durch die Landnutzer*innen geachtet.

\section{Auswahl des Fallstudienlands}

Vier Faktoren waren ausschlaggebend für die Auswahl des Fallstudienlands. Erstens, eine hohe Relevanz traditioneller Autoritäten in den Bereichen Landverwaltung und Konfliktmanagement. Zweitens, die deutlich unterschiedliche Ausgestaltung traditioneller Herrschaft innerhalb des gleichen Länderkontexts. Drittens, ein zahlen- und flächenmäßig großes Ausmaß von land grabbing. Und viertens, die Existenz von Konflikten um Land im Kontext von land grabbing in denen traditionelle Autoritäten als Konfliktakteure in Erscheinung treten.

Der aktuelle Stand der Forschung zeigt, dass traditionelle Autoritäten in vielen Ländern Sub-Sahara Afrikas eine bedeutende Rolle in der Landverwal- 
tung einnehmen (Stacey 2015: 26). Die staatliche Anerkennung traditioneller Autoritäten als Verwalter*innen von Land variiert in den unterschiedlichen Ländern erheblich. In vielen Ländern Subsahara-Afrikas üben sie diese Aufgabe in der alltäglichen Praxis aus, ohne eine rechtliche Grundlage und ohne offizielle Anerkennung oder Legitimierung durch den Staat. Ausnahmen diesbezüglich bilden unter anderem Ghana, Mosambik und Südafrika (Ubink 2008c), wo postkoloniale Regierungen die Stellung traditioneller Autoritäten $\mathrm{ab}$ den frühen 1980er Jahren formalisiert haben und traditionelle Herrschaft durch die verfassungsrechtliche Anerkennung staatlich legitimiert ist. In Ghana ist die formale Anerkennung traditioneller Herrschaft durch die Verfassung von 1992 gewährleistet (vgl. Kasanga/Kotey 2001). Ghana und Südafrika heben sich von anderen Ländern Subsahara-Afrikas insbesondere dadurch ab, dass traditionelle Autoritäten einen großen Einfluss in öffentlichen Angelegenheiten haben.

Die Relevanz traditioneller Autoritäten in den Bereichen Landverwaltung und Konfliktmanagement ergibt sich nicht alleine aus der staatlichen Anerkennung und Formalisierung traditioneller Herrschaft, sondern auch aus der Ausgestaltung der alltäglichen Praxis. Im Unterschied zu Ländern wie Mosambik und Südafrika, genießen traditionelle Autoritäten in Ghana neben der formalen Anerkennung auch eine besondere Anerkennung und Akzeptanz seitens der Bevölkerung, wie Umfragen des Afrobarometers zeigen (Logan 2013). Sowohl im Bereich der Landvergabe wie auch des Konfliktmanagements ist ihr Einfluss besonders groß. Die Umfrageergebnisse zeigen, dass traditionelle Autoritäten in der Landvergabe und im Konfliktmanagement nach Wahrnehmung der Befragten die Hauptverantwortung tragen. Die Studie berücksichtigt 18 Länder. Von diesen ist Ghana das einzige Land, das sich in beiden Bereichen unter den ersten drei Ländern mit den höchsten Umfragewerten für die Zuständigkeit traditioneller Autoritäten befindet. Die Befragten gaben an, dass traditionellen Autoritäten in Ghana im Vergleich zu staatlichen Akteuren in der Landvergabe sowie im Konfliktmanagement den größten Einfluss ausüben. Im Bereich der Landvergabe schrieben die Befragten traditionellen Autoritäten 70 Prozent der Zuständigkeit zu. Damit befindet sich Ghana an der Spitze aller Umfrageländer (Logan 2013: 361). Die folgenden Grafiken veranschaulichen wie die Öffentlichkeit die Zuständigkeit traditioneller Autoritäten in der Lösung von Konflikten (Abb. 1) und der Vergabe von Land (Abb. 2) in Ghana und 17 anderen Ländern Subsahara-Afrikas wahrnimmt. 
Abbildung 1: Wahrgenommene Zuständigkeitsverteilung zwischen Lokalregierung und traditionellen Autoritäten in der Konfliktlösung

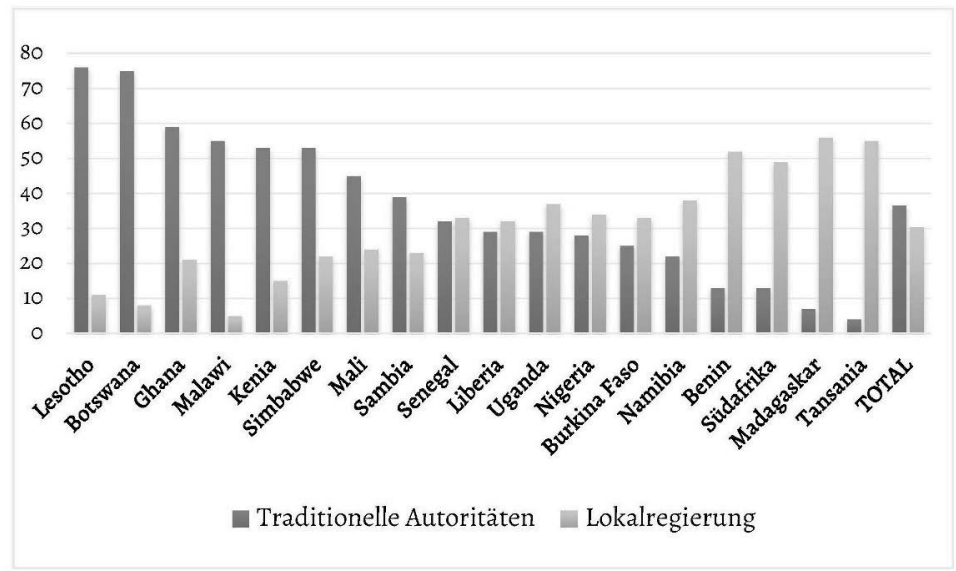

Quelle: Logan 2013: 361, eigene Darstellung, Angaben in Prozent

Gleichzeitig hat sich Ghana seit Beginn der 2000er Jahre zu einem beliebten Zielland für die Umsetzung großflächiger inter- und multinationaler agrarindustrieller Projekte entwickelt (Schoneveld/German 2014). Seit langer Zeit gilt das Land als politisch stabil, verfügt über eine vergleichsweise gute Infrastruktur und weist mit dem höchsten Bruttoinlandsprodukt pro Kopf in Westafrika eine relativ solide Wirtschaft auf. Der Agrarsektor erwirtschaftet ca. 20 Prozent des gesamten BIPs und beschäftigt die Hälfte aller Erwerbstätigen (The World Factbook 2016). Zudem hat Ghana durch eine lange Geschichte des Goldbergbaus und der Plantagenwirtschaft Erfahrungen mit internationalen Investoren. Laut Weltbank ist Ghana aufgrund der stabilen politischen Lage, günstiger steuerlicher Rahmenbedingungen und einer hohen Rechtssicherheit eines der investorenfreundlichsten Länder Afrikas (FIAN 2010: 1; Schoneveld/German 2014: 188). Zudem verfügt das Land über große Vorkommen an Rohstoffen (unter anderem Gold, Diamanten und Öl) sowie über fruchtbares Land (Annan et al. 2013: 1). Unter Berücksichtigung aller Landtransaktionen mit einer Fläche von mehr als 200 Hektar haben Investoren in Ghana, laut Land Matrix, im Zeitraum von 2000 bis 2015 Pachtverträge 
Abbildung 2: Wahrgenommene Zuständigkeitsverteilung zwischen Lokalregierung und traditionellen Autoritäten in der Landvergabe

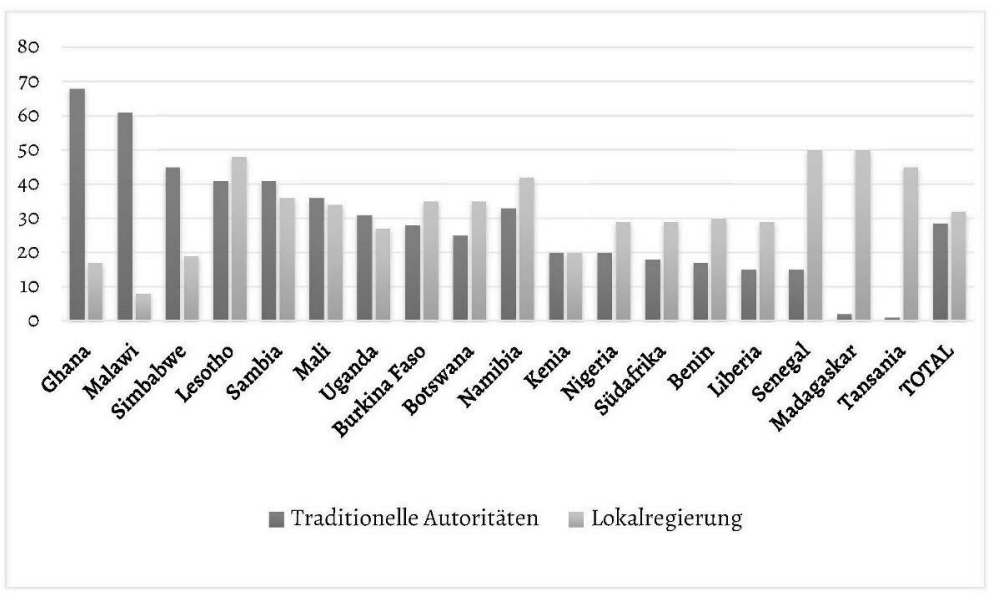

Quelle: Logan 2013: 361, eigene Darstellung, Angaben in Prozent

über mehr als eine Million Hektar Land unterzeichnet (The Land Matrix 2019). Das entspricht dem Vierfachen der Fläche Luxemburgs. Damit liegt Ghana im Vergleich mit anderen Ländern Subsahara-Afrikas vom Flächenausmaß nur hinter Mosambik und Äthiopien.

Wie auch in anderen Ländern treten im Verlauf der Verhandlungen großflächiger Landvergabe und während der Implementierung der Projekte in Ghana häufig Konflikte auf: Konflikte zwischen unterschiedlichen Gruppen von Landnutzer*innen, Konflikte im Zusammenhang mit illegalen Verkäufen von Land oder der Intransparenz der Verhandlungen, und Konflikte zwischen unterschiedlichen Nutzer*innengruppen wie Kleinbäuerinnen und -bauern und mobilen Viehzüchter*innen (Tsikata/Yaro 2011). Traditionelle Autoritäten sind zentrale Akteure in diesen Konflikten. Spätestens seit Beginn der 200oer Jahre gehören sie in Ghana zu den wichtigsten Verhandlungspartner*innen in Landtransaktionen (Cotula et al. 2014). Vor allem im Verhältnis zu staatlichen Institutionen haben traditionelle Autoritäten seither ihre Macht im Bereich der Landvergabe maßgeblich ausgeweitet. Während in vielen anderen Ländern staatliche Institutionen aktiv an Landvergabeprozessen beteiligt sind, 
spielen sie in Ghana nur eine untergeordnete Rolle und agieren zunehmend nur noch als bestätigende Instanzen. Traditionelle Autoritäten hingegen haben aufgrund der gesetzlichen Anerkennung von traditionellem Recht und ihrer uneingeschränkten Zuständigkeit in der Landverwaltung (Republic of Ghana 1992: Art. 267(1)) relativ freie Hand in den Verhandlungen mit Investoren. Zugleich sind sie laut Verfassung verpflichtet, als Treuhänder*innen die Interessen ihrer Gemeinschaft zu vertreten (Republic of Ghana 1992: Art. 36(8)). Ihnen obliegt es, die Zugangsrechte der Landnutzer*innen zu schützen, im Fall des Verlusts von Land für angemessene Entschädigungszahlungen zu sorgen, Kleinbäuerinnen und -bauern in die Verhandlungen miteinzubeziehen und ihnen die Möglichkeit des Widerspruchs oder der Überprüfung von Vereinbarungen zu geben (Elhadary/Obeng-Odoom 2012; Vermeulen/Cotula 2010: 907). In der Praxis kommen sie dieser Aufgabe oftmals jedoch nicht nach, sondern handeln nach eigenem Interesse und ohne die Zustimmung der Landnutzer*innen. Aufgrund ihrer zentralen und ambivalenten Rollen als Verhandlungspartner*innen sowie Treuhänder"innen in der Verhandlung großflächiger Landtransaktionen sind traditionelle Autoritäten in Ghana zunehmend auch Konfliktakteure. Welche Rolle sie in diesen Konflikten spielen, ist in der Forschung bislang kaum untersucht worden (Boamah 2014a: 407). Mit Blick auf die besonders starke Stellung traditioneller Autoritäten und das im Verhältnis hohe zahlen- und flächenmäßig Ausmaß an land grabbing Fällen ist Ghana als Fallstudienland nicht repräsentativ für den gesamten Kontinent, sondern vielmehr ein trouble case, wie Berry (2017: 114f.) es fasst. Jason Seawright und John Gerring sprechen diesbezüglich von einem extremen Fall (extreme case) (Seawright/Gerring 2008:301), einem Fall der sich hinsichtlich zentraler analytischer Dimensionen als besonders stark oder schwach erweist. Als extremes Fallstudienland eignet Ghana sich besonders gut, um eine größtmögliche Menge an Informationen zu einem bisher noch wenig erforschten Phänomen zu erlangen (Flyvbjerg 2011: 16).

\section{Auswahl der Fälle}

Ein Fall ist in meiner Studie ein empirisch beobachtbarer Konflikt um Zugang zu Land, in dem traditionelle Autoritäten als zentrale Konfliktakteure auftreten. Folgende drei Kriterien bestimmten die Auswahl meiner Fälle. Erstens sollten die Fälle sich in Regionen befinden, in denen sich die großflächige agarindustrielle Umnutzung von Land seit Mitte der $2000 e r$ Jahre maßgeblich ausgeweitet hat. Zweitens sollten in diesen Regionen im Zusammenhang 
mit land grabbing vermehrt Konflikte auftreten. Und drittens sollten die Fälle in zwei Regionen liegen, in denen sich die Ausgestaltung der traditionellen Herrschaft unterscheidet. Jenseits dieser Kriterien war der Anspruch an die Fallauswahl, eine größtmögliche Vergleichbarkeit zu gewährleisten. Unter Berücksichtigung dieser Kriterien fiel die Auswahl der Fälle auf die Konflikte um das ScanFarm-Projekt in der Ashanti Region und das BioFuel Africa-Projekt in der Northern Region. Als typical cases (Gerring 2007: 91) sind sie repräsentativ für andere Konflikte, die im Zusammenhang mit land grabbing in Ghana und darüber hinaus entstanden. Dies bezieht sich insbesondere auf den Umsetzungszeitpunkt der Projekte, den Zweck der Umnutzung des Lands, die intendierte Größe der gepachteten Fläche sowie die am Konflikt beteiligten Akteure. In Bezug auf die Ausgestaltung der traditionellen Herrschaft in beiden Forschungsregionen handelt es sich um diverse cases (Gerring 2007: 97f.). Die Mischung aus typical und diverse case leitet sich aus meinem Erkenntnisinteresse sowie meinen theoretischen Vorannahmen ab. Ich möchte Aussagen zum Handeln traditioneller Autoritäten in Konflikten im Kontext von land grabbing treffen, deren Reichweite über die von mir untersuchten Fälle hinausgehen. Aus analytischer Perspektive eignen sich diesbezüglich Fälle, die ein breites Spektrum anderer Fälle repräsentieren, also typische Fälle. Gleichzeitig ist meine Vorannahme, dass Unterschiede in der traditionellen Herrschaft, und damit verbunden das unterschiedliche Handeln traditioneller Autoritäten, Auswirkungen auf die Entstehung und den Verlauf von Konflikten um Zugang zu Land haben. Für die Analyse setzt dies voraus, dass ich mindestens zwei Fälle untersuche, in denen sich die traditionelle Herrschaft unterscheidet. Als Forschungsdesign leitet sich daraus die diverse-case-Strategie $a b$.

$\mathrm{Ab}$ Mitte der 2000er Jahre kam es in vielen Ländern Subsahara-Afrikas und insbesondere in Ghana - zur Ausweitung des großflächigen Anbaus von Energiepflanzen für die Produktion von Agrartreibstoffen. Insbesondere der Anbau von Jatropha erfuhr einen regelrechten Boom. In diesem Kontext kam es vielerorts zu Konflikten um das für den Anbau von Jatropha vorgesehene Land (Campion/Acheampong 2014; Hunsberger 2010; Schrader 2014; von Maltitz et al. 2014). Innerhalb Ghanas variierte die räumliche Ausbreitung des Jatropha-Anbaus erheblich. Im südlichen Ghana konzentrierte sie sich hauptsächlich auf die Regionen Brong-Ahafo und Ashanti und im nördlichen Teil des Landes auf die Regionen Upper West, Northern Region und Upper 
East $^{1}$ (Yirrah 2012). Mittlerweile ist der Jatropha-Boom vorüber. Ehemalige Jatropha-Projekte sind entweder gescheitert oder die für den Jatropha-Anbau eingeplanten Flächen dienen nun dem Anbau anderer Nutzpflanzen. Die Konflikte dauern jedoch in vielen Fällen an. Die Projekte ScanFarm und BioFuel Africa sind repräsentativ für dieses Phänomen und zeichnen sich darüber hinaus durch eine gute Vergleichbarkeit aus. Beide Projekte wurden zwischen 2005 und 2008 von norwegischen Investoren mit dem Ziel des Jatropha-Anbaus initiiert, waren jedoch wie zahlreiche andere Projekte erfolglos mit diesem Vorhaben. ScanFarm produziert seit 2009 Mais und in geringem Umfang unterschiedliche andere Pflanzen für den lokalen Markt und den Export. BioFuel Africa scheiterte 2011 gänzlich. Seither liegen die ursprünglich für den Jatropha-Anbau gepachteten Flächen brach und es ist unklar, wer aktuell über die Nutzungsrechte verfügt. In beiden Konflikten zählen traditionelle Autoritäten zu den zentralen Konfliktakteuren, wobei sich die Ausgestaltung der traditionellen Herrschaft zwischen der Ashanti Region und der Northern Region unterscheidet. In der Ashanti Region sind traditionelle Autoritäten historisch betrachtet und bis in die heutige Zeit besonders einflussreich. Sie sind in stark hierarchischen Strukturen organisiert, die in großem Maß auf die Kolonialzeit zurückgehen. Insbesondere traditionelle Autoritäten in hohen Positionen gehören in der Regel der nationalen Elite an und verfügen über große (internationale) Netzwerke in Politik und Wirtschaft. Im Vergleich dazu unterscheiden sich die drei nördlichen Regionen in der Ausgestaltung der traditionellen Herrschaft. Traditionelle Autoritäten in den nördlichen Regionen sind in der Regel weniger stark in (inter)nationale Netzwerke eingebunden und verfügen im Vergleich über weniger Ressourcen. Die Vergabe von Land liegt jedoch in beiden Regionen gleichermaßen in der Verantwortung traditioneller Autoritäten. Im Dagbon-Königreich, der Forschungsgegend in der Northern Region, haben die paramount chiefs jedoch eine Rechenschaftspflicht gegenüber dem Ya Naa, dem König der Dagbon und der höchsten traditionellen Autoritäten. In der Ashanti Region hingegen können die paramount chiefs Entscheidungen bezüglich der Vergabe von Land ohne Absprache mit dem Asantehene, dem König der Ashanti, beschließen. 


\subsection{Erhebung der Daten}

Die zentralen Methoden der Datenerhebung für meine Studie sind teilstrukturierte Leitfadeninterviews und Gruppendiskussionen. Im Laufe von drei Feldforschungsphasen in den Zeiträumen von November 2015, März bis Juni 2016 und Februar bis März 2017 habe ich insgesamt 46 Interviews mit 52 Personen und sieben Gruppendiskussionen mit insgesamt rund 170 Personen geführt. Hinsichtlich der Auswahl der Interviewpartner*innen verfolgte ich den Anspruch, ein möglichst großes Spektrum an für die Fälle relevanter Akteure abzubilden. Anhand einer umfassenden Literaturauswertung sowie im Verlauf des ersten explorativen Feldforschungsaufenthalts 2015 identifizierte ich sechs zentrale Akteursgruppen: Mitarbeiter*innen staatlicher Institutionen, Mitarbeiter*innen traditioneller (Landrechts-)Institutionen, traditionelle Autoritäten, Landnutzer*innen, Unternehmensakteure sowie zivilgesellschaftliche Akteure. Interviewpartner*innen waren dementsprechend Mitarbeiter*innen staatlicher Institutionen auf nationaler, regionaler und Distriktebene, Repräsentanten der CLS (traditionelle Landrechtsinstitution), Vertreter des National House of Chiefs sowie des Regional House of Chiefs der Northern Region, traditionelle Autoritäten und ihre Berater (elders) sowie Landnutzer*innen der betroffenen Gegenden, leitende Angestellte der Unternehmen und Mitarbeiter*innen zivilgesellschaftlicher Organisationen. Teilnehmende an den Gruppendiskussionen waren die durch die agrarindustriellen Projekte betroffenen Landnutzer*innen und traditionellen Autoritäten der Dörfer Nsonyameye und Dukusen (ScanFarm), Jimle, Kpachaa, Parachanayilli, Tuya, Changolinaaya und Jashei sowie die elders des paramount chiefs von Tijo (BioFuel Africa). Bei der Auswahl der Interviewpartner*innen aus der Gruppe der Landnutzer*innen habe ich auf eine ausgeglichene Verteilung hinsichtlich der Kategorien Herkunft, Alter, Geschlecht und Art des Landzugangs geachtet. In beiden Fällen gab es Landnutzer*innen, die den Zugang zu ihrem Land über eine traditionelle Autorität erhalten hatten und andere, die sich als Nachkommen derjenigen bezeichneten, die das Land als erste bestellt haben. Ihr Zugang zu Land unterliegt somit nicht der Kontrolle durch traditionelle Autoritäten. Darüber hinaus gab es Landnutzer*innen, die ursprünglich aus der Gegend waren und andere die zugezogen waren. In den Einzelinterviews erwies es sich schwierig mit jungen Menschen gleich welchen Geschlechts zu sprechen. In der Regel waren sie aufgrund ihrer Arbeit stark eingebunden und konnten nur wenig Zeit für ein Interview aufbringen. Darüber hinaus zogen sie es meistens vor, den älteren Menschen aus ihrer Familie das Wort 
zu überlassen, da sie selbst ihren Zugang zu Land über Letztere erhielten. In den wenigen Interviews die ich mit jungen Menschen führen konnte, stellte sich letztendlich aber auch heraus, dass sie zu dem Konflikt in Zusammenhang mit der Vergabe des Lands an das Unternehmen nur wenig sagen konnten und mich immer wieder an ältere Menschen aus ihrer Familie verwiesen, die ihrer Meinung nach über ein umfassenderes Wissen verfügten.

Im Folgenden erläutere ich zunächst meine Beweggründe für die Wahl teilstrukturierter Leitfadeninterviews und Gruppendiskussionen als Erhebungsmethoden und lege dar, wie ich bei der Entwicklung der Leitfäden vorgegangen bin. Im Anschluss daran gehe ich näher darauf ein, wie ich mir Zugang zum Feld verschafft habe, wie ich in der Durchführung der Interviews vorgegangen bin und diskutiere die jeweiligen damit verbundenen Herausforderungen. Schließlich erörtere ich meinen Anspruch, mittels Iteration und Triangulation die Qualität der erhobenen Daten abzusichern.

\section{Teilstrukturierte Leitfadeninterviews und Gruppendiskussionen}

Das teilstrukturierte Leitfadeninterview zeichnet sich durch die Qualität »so offen wie möglich, so strukturierend wie nötig« (Helfferich 2014:560) zu sein, aus. Damit ist es als Erhebungsmethode gut geeignet, um bislang wenig erforschten Phänomenen mit großer Offenheit zu begegnen. Für die Entwicklung meiner Leitfäden habe ich mich an Cornelia Helfferichs (2011: 186) Ausführungen orientiert. Die Struktur dieses Leitfadens zeichnet sich dadurch aus, dass sie narrativ ausgelegt ist und den Interviewten dadurch die Möglichkeit gibt, ihre eigene Perspektive darzustellen, eigene Schwerpunkte zu setzen und auf Themenbereiche einzugehen, die der Leitfaden nicht vorgibt. Gleichzeitig ermöglicht die offene und flexible Teilstrukturierung Interviewenden, forschungsrelevante Themenbereiche nicht aus dem Blick zu verlieren und gezielte Nachfragen zu stellen, wenn Interviewte bestimmte relevante Themen selbst nicht ansprechen oder sich das Interview in eine Richtung entwickelt, die forschungsirrelevant ist. In Anlehnung an das von Helfferich entwickelte Schema habe ich die Leitfäden je nach Akteursgruppe in vier bis fünf große Themenkomplexe strukturiert, die ich aus der vorherigen Literaturauswertung und mit Blick auf mein Erkenntnisinteresse entwickelt habe. Jeder Themenkomplex beginnt mit einer Erzählaufforderung. Diese Frage gibt an, in welche Richtung das Forschungsinteresse geht, ist jedoch so offen gestellt, dass sie die Interviewpartner*innen ermuntern soll, ihre eigene Perspektive auf das jeweilige Thema darzustellen. Um forschungsrelevante 
Punkte nicht aus dem Blick zu verlieren, enthält jeder Themenkomplex eine Anzahl an Memos. Dies sind Themen, die idealerweise durch die Interviewten selbst thematisiert werden sollten. Ist dies nicht der Fall, können sie im Verlauf des Interviews an geeigneter Stelle als gezielte Nachfrage integriert werden. Den Abschluss des Interviewleitfadens bildet die Frage nach weiteren relevanten Themen, die im Verlauf des Interviews noch nicht angesprochen wurden. Ziel dieser Frage war es, Raum für Themen zu geben, die im bisherigen Forschungsprozess fehlten, von den Beforschten selbst jedoch als wichtig erachtet werden. Mit Blick auf Themen, welche die Befragten selbst einbrachten und damit zusammenhängenden neuen Erkenntnissen habe ich den Interviewleitfaden im Forschungsprozess immer wieder angepasst und ergänzt.

Den Leitfaden habe ich sowohl für die Einzelinterviews als auch in Gruppendiskussionen verwendet. Gruppendiskussionen habe ich ausschließlich mit den von den agrarindustriellen Projekten betroffenen Landnutzer*innen geführt. In diesem Kontext eigneten sich besonders Gruppendiskussionen als Methode gut, um die kollektive Meinung der Gruppe in Bezug auf den Landnutzungswandel, seine Folgen und Konflikte, die damit in Zusammenhang stehen, herauszuarbeiten. In Gruppendiskussionen steht nicht die gleichzeitige Befragung mehrerer Personen im Vordergrund, sondern die Interaktion der Gruppenmitglieder (Bohnsack 2009: 360; Vogl 2014: 581). Um eine möglichst hohe Informationsdichte $\mathrm{zu}$ erlangen, hätte ich es aus methodischer Sicht vorgezogen, Fokusgruppendiskussionen mit Realgruppen - sowie künstlichen Gruppen von maximal 12 Teilnehmer*innen durchzuführen (Vogl 2014: 584). Realgruppen sind Gruppen, die das reale soziale Gefüge der Gruppe aus der die Interviewpartner*innen stammen, abbilden. In Bezug auf die Landnutzer*innen hätte ich entsprechend innerhalb der Gruppe auf eine Varianz bezüglich Alter und Geschlecht geachtet. Künstliche Gruppen sind Gruppen, die hinsichtlich einer oder mehrerer Kategorien homogen sind. So hätte ich gerne Gruppen aus jungen Frauen, älteren Frauen, Frauen gemischten Alters, jungen Männern, älteren Männern und Männern gemischten Alters gebildet. Das war in der praktischen Durchführung jedoch nicht möglich, da es die Landnutzer"innen in den - meist sehr kleinen Dörfern bevorzugten, gemeinsam interviewt zu werden. 


\section{Feldzugang und Durchführung der Interviews}

Die Grundvoraussetzung für die erfolgreiche Durchführung einer Fallstudie ist der Zugang zum Feld. Diesen erarbeitete ich mir sukzessiv im Verlauf meiner drei Forschungsaufenthalte. Ziel der ersten Forschungsreise war die Sondierung des Forschungsfelds, die Vernetzung mit relevanten Akteuren und die Fallauswahl. Während dieses einmonatigen Aufenthalts im November 2015 traf ich Mitarbeiter*innen zivilgesellschaftlicher Organisationen und Wissenschaftler*innen, die sich mit dem Thema land grabbing sowie damit einhergehenden Konflikten in Ghana auseinandergesetzt hatten. Mein Anliegen war es, ein Kontaktnetzwerk aufzubauen, das hilfreich für die Fallauswahl sowie für den Zugang zum Feld sein würde. Mein erster Zugangspunkt war die NGO Action Aid International Ghana, auf die ich durch die vorherige Literaturauswertung aufmerksam geworden war. Diese stellte den Kontakt zu einem Mitarbeiter des Food Security Policy Advocacy Network (FoodSPAN) her. Gemeinsam hatten beide intensiv zu Konflikten geforscht, die im Rahmen steigender internationaler Investitionen in den Anbau von Energiepflanzen zur Gewinnung von Agrartreibstoffen auftraten und zahlreiche Publikationen dazu veröffentlicht. Mein zweiter Zugangspunkt war die University of Ghana. Dort tauschte ich mich mit drei Wissenschaftler"innen aus, die zahlreiche Artikel zu land grabbing, Konflikten um Land und traditioneller Herrschaft publiziert hatten. Diese bat ich jeweils um weitere Kontaktpersonen, die für mein Forschungsvorhaben relevant sein könnten. So erarbeitete ich mir über das Schneeballverfahren kontinuierlich weitere Kontakte, die mich schließlich maßgeblich in der Auswahl meiner Fälle unterstützten. Als ersten Fall identifizierte ich so das ScanFarm Projekt. Auch während meines zweiten Aufenthalts von Februar bis Juni 2016 hatte ich die Möglichkeit, Kontakte zu Wissenschaftler*innen und Mitarbeiter"innen Zivilgesellschaftlicher Organisationen zu knüpfen. Durch zahlreiche Diskussionen mit unterschiedlichen Gesprächspartner"innen und weitere Literaturrecherche identifizierte ich den zweiten Fall, das BioFuel Africa Projekt.

Ich begann mit der Datenerhebung in Accra, der Hauptstadt Ghanas. Ziel war es, zunächst allgemeine Informationen zu Konflikten im Kontext von land grabbing, traditioneller Herrschaft, Funktionsweise und Zusammenspiel des traditionellen und staatlichen Landrechtssystems sowie zu Entwicklungsund Investitionsstrategien im Landwirtschaftssektor zu erheben. In diesem Rahmen führte ich zwischen 2015 und 2017 zwei Interviews mit Mitarbeiter*innen zivilgesellschaftlicher Organisationen und vier Interviews mit Mit- 
arbeitern staatlicher Stellen. Die zweite Etappe der Feldforschung war Kumasi, die Regionalhauptstadt der Ashanti Region. Dort realisierte ich zunächst ein Interview mit dem Mitarbeiter einer NGO, der über hilfreiche Informationen zum ScanFarm Projekt verfügte und mir diesbezüglich weitere Kontakte vermitteln konnte. Zwischen 2016 und 2017 führte ich in Kumasi des Weiteren drei Interviews mit Mitarbeiter*innen staatlicher Stellen, die in die Vergabe des Lands an ScanFarm involviert waren, sowie mit einem Mitarbeiter des Asantehenes Land Secretariats, der regionalen traditionellen Landrechtsinstitution und einem Mitarbeiter des National House of Chiefs. Die dritte Etappe meiner Feldforschung war Agogo, die Hauptstadt des Asante Akyem North Districts, wo sich das ScanFarm Projekt befindet. Dort führte ich 2016 und 2017 zwei Interviews mit dem CEO von ScanFarm und zwei mit Mitarbeitern traditioneller (Landrechts-)Institutionen. Außerdem war es mir möglich zwei Interviews mit staatlichen Akteuren auf Bezirksebene zu realisieren. Die Sprache dieser Interviews war ausschließlich Englisch mit Ausnahmen desjenigen mit den Mitarbeitern traditioneller (Landrechts-)Institutionen, die es vorzogen auf Twi zu kommunizieren. Bei der Übersetzung hatte ich Hilfe durch einen Forschungsassistenten.

Den Großteil meiner Interviews in Agogo und den durch das ScanFarm-Projekt betroffenen Dörfern führte ich mit den traditionellen Autoritäten der jeweiligen Dörfer und den Landnutzer*inenn. Den Zugang zu diesen Interviewpartner*innen erschloss ich mir über unterschiedliche Wege. Den Erstkontakt zu den chiefs von Dukusen, Nsonyameye und Baamaa sowie $\mathrm{zu}$ einigen Landnutzer*innen stellte ein Mitarbeiter des Department of Agriculture her, der für dessen Community-Arbeit zuständig war und einen guten Zugang zu den Landnutzer*innen hatte. Er leistete auch die organisatorische Vorarbeit für eine Gruppendiskussion. Weitere Kontakte zu Landnutzer*innen erhielt ich über das Schneeballverfahren. So entstand auch der Kontakt zu den Agogoman mma kuo (weltweite Bürger*innen von Agogo). Die Agogoman mma kuo ist ein zivilgesellschaftliches Netzwerk engagierter Bewohner*innen von Agogo, das bereits vor der Implementierung des ScanFarm Projekts existierte und sich mittlerweile für die Interessen einiger Landnutzer*innen einsetzt. Insgesamt realisierte ich 14 Interviews mit Landnutzer*innen. Bis auf ein Interview, das in englischer Sprache stattfand, führte ich diese Interviews auf Twi mit einer Übersetzung durch meinen Forschungsassistenten.

Für die Datenerhebung zum Konflikt um BioFuel Africa konnte ich den Leiter der NGO Northern Accelerated Intervention for Development als Forschungsas- 
sistenten gewinnen, der mit dem Umfeld und dem lokalen Verhaltenskodex vertraut war. Die Zusammenarbeit mit ihm vereinfachte den Zugang zum Feld. Er vermittelte mir Kontakte zu einigen staatlichen Stellen, zum CLS in der Regionalhauptstadt Tamale sowie zu einem leitenden Angestellten des Unternehmens. Darüber hinaus leistete er die organisatorische Vorarbeit für ein Interview und insgesamt sechs Gruppendiskussionen mit traditionellen Autoritäten und Landnutzer*innen in sieben Dörfern der Gegend des BioFuel Africa Projekts. Mit einigen anderen staatlichen Stellen und dem Regional House of Chiefs vereinbarte ich eigenständig Interviewtermine. Insgesamt realisierte ich so während der Feldforschungsaufenthalte 2016 und 2017 fünf Interviews mit Mitarbeiter*innen regionaler staatlicher Institutionen, zwei Interviews mit Repräsentanten formalisierter traditioneller Institutionen auf regionaler Ebene und ein Interview mit einem Angestellten des Unternehmens. Diese Interviews führte ich allein und auf Englisch durch. Das Interview mit zwei Landnutzern aus der betroffenen Gegend sowie die sechs Gruppendiskussionen mit Landnutzer*innen und traditionellen Autoritäten fanden auf Dagbani mit Übersetzung ins Englische durch meinen Forschungsassistenten statt.

In allen bis auf zwei Fällen willigten die Interviewpartner*innen in die Audioaufzeichnung des Interviews ein. Zu den anderen Interviews sowie den Gruppendiskussionen, die nicht für eine Audioaufzeichnung geeignet sind, fertigte ich Gesprächsprotokolle an.

\section{Qualitätssicherung der Daten durch Iteration und Triangulation}

Die Aussagen von Interviewpartner*innen spiegeln immer ihre persönliche Wahrnehmung und Einschätzung wider und können daher als »subjektive Wahrheit« verstanden werden, »die für den spezifischen Erzählaugenblick gültig ist« (Helfferich 2014: 561). Um dennoch Vertrauen in die eigenen Daten $\mathrm{zu}$ entwickeln, habe ich in meiner Forschung Iteration und DatenTriangulation angewandt. Iteration bedeutet, vielen unterschiedlichen Interviewpartner"innen die gleichen Fragen zu stellen, bis ein gewisser Sättigungsgrad an Informationen erreicht ist (Lund 2014: 226f.). Triangulation bedeutet die empirischen Daten mit anderen Quellen abzugleichen, um so mindestens zwei Sichtweisen auf den gleichen Untersuchungsgegenstand $\mathrm{zu}$ erhalten (Yin 2009). Während Iteration zur Bestätigung von Aussagen nützlich sein kann, eignet sich Triangulation zur Generierung weiterer Erkenntnisse (Flick 2014: 419). Daten-Triangulation »kombiniert Daten, die 
verschiedenen Quellen entstammen und zu verschiedenen Zeitpunkten, an unterschiedlichen Orten oder bei verschiedenen Personen erhoben werden« (Flick 2014: 418). So habe ich im Vorfeld und im Verlauf der empirischen Datenerhebung eine umfassende Analyse wissenschaftlicher Literatur zu meinem Forschungsfeld durchgeführt und habe Gesetzestexte, Zeitungsartikel, Blog-Beiträge, Berichte zivilgesellschaftlicher Organisationen, graue Literatur und Unternehmensberichte ausgewertet. Die Berücksichtigung und Auswertung zusätzlicher Quellen in Ergänzung zur eigenen Forschung stärkt die Plausibilität der eigenen empirischen Daten, dient aber nicht ihrer abschließenden Validierung (Lund 2014: 227). Sie können jedoch hilfreich sein, um die Bedeutung von Aussagen zu überprüfen, unterschiedliche Perspektiven auf das gleiche Phänomen aufzudecken, und die Reproduzierbarkeit einer Beobachtung oder Interpretation $\mathrm{zu}$ belegen (Stake 2003: 148).

\subsection{Reflexion des methodischen Vorgehens}

Im Verlauf der Forschung habe ich einige Herausforderungen hinsichtlich des methodischen Vorgehens identifiziert. Diese reflektiere ich im Folgenden und gehe auf meinen Umgang mit ihnen ein. Zentrale Herausforderungen waren mit der Übersetzung der Interviews verbunden, mit der fehlenden Bereitschaft der Partizipierenden, Einzelinterviews zu geben und mit ungleichen Redeanteilen der einzelnen Akteure in Gruppendiskussionen. Nicht zuletzt erwies sich auch der Vergleich der beiden Fälle als herausfordernd.

Während Mitarbeiter*innen staatlicher Stellen und zivilgesellschaftlicher Organisationen in der Regel sehr gut Englisch sprechen, bevorzugte ein Großteil der Landnutzer*innen und traditionellen Autoritäten die Kommunikation in ihrer jeweiligen Erstsprache. In der Ashanti Region war dies Twi, in der Northern Region Dagbani. Für Interviews und Gruppendiskussionen, die ich mit diesen Akteuren führte, war daher immer eine Übersetzung nötig. Diese beeinflusst immer auch die Gesprächsatmosphäre und birgt das Risiko, sich hemmend auf den Gesprächsfluss auszuwirken. Die meisten Interviewten sind jedoch mit diesem Umstand vertraut und äußerten sich sehr offen. Sonstige Schwierigkeiten, die mit der Übersetzung verbunden sind, habe ich im Vorfeld detailliert mit meinen Forschungsassistenten besprochen. Besonderen Wert habe ich darauf gelegt, dass meine Fragen und die Antworten der Interviewten immer im O-Ton übersetzt wurden und dass die Antworten 
nicht in zusammenfassender Form wiedergegeben werden, damit auch die Argumentationsstruktur nachvollziehbar bleibt.

Die Interviews und Gruppendiskussion, die ich mit den Landnutzer*innen in der Ashanti Region führte, zeichneten sich durch eine große Offenheit von Seiten der Interviewpartner*innen aus, die mir bereitwillig Auskunft zu meinen Fragen gaben. In der Northern Region erwies sich die Forschungssituation als schwieriger. Im Allgemeinen standen mir die meisten Landnutzer*innen zunächst sehr viel skeptischer und verhaltener gegenüber. Um eine Vertrauensbasis zu schaffen, berichtete ich ausführlich über meine Forschung und gerantierte ihnen ihre Anonymität. Das entspannte die Atmosphäre maßgeblich. Letztendlich entstand so eine größere Offenheit seitens der Landnutzer*innen und es stellte sich heraus, dass einige von ihnen bereits schlechte Erfahrungen mit Forscher*innen gemacht hatten. Vor allem waren sie aber nicht bereit Einzelinterviews zu geben. Da sie in sehr kleinen Dörfern zusammenleben, zogen sie es vor, dass alle Dorfbewohner*innen im Rahmen von Gruppendiskussionen am Forschungsprozess teilhaben konnten. Auf diese Weise wollten sie gewährleisten, dass sie alle dieselben Informationen erhielten und gleichermaßen die Möglichkeit bekamen, ihre Meinung zu äußern. Dennoch variierten in einigen von mir geführten Gruppendiskussionen die Redeanteile der teilnehmenden Personen mitunter beträchtlich, worauf ich mich jedoch bereits im Vorfeld eingestellt hatte. Denn Gruppendiskussionen sind immer durch Machtverhältnisse unter den Teilnehmenden strukturiert, die bestimmen, wer sich in welchem Umfang zu Wort meldet, wer in der Lage ist, die eigene Meinung anderen gegenüber offenzulegen und zu vertreten. Problematisch an Gruppendiskussionen ist also, dass die öffentliche Diskussion die Äußerung individueller, eventuell von der Mehrheit abweichender Meinungen, verhindern kann (Vogl 2014: 582). Insbesondere Frauen und junge Menschen meldeten sich in diesen Fällen weniger zu Wort. Traditionelle Autoritäten und ältere Männer dominierten die Diskussion in der Regel. Wenn die Gesprächsatmosphäre und Haltung der anderen Teilnehmenden es zuließen, habe ich gegen Ende der Gruppendiskussion explizite Erzählaufforderungen an Gruppen mit geringem Redeanteil gerichtet. Um Situationen wie diese in der Zukunft zu umgehen, könnte eine Möglichkeit sein, aus der großen Gruppe Kleingruppen zu bilden und diese in weibliche, männliche und gemischte Teilnehmer*innen zu unterteilen. Vor allem in Frauengruppen könnte es darüber hinaus sinnvoll sein, eine Übersetzerin zu engagieren, da die Anwesenheit von Männern in einigen Kontexten zu einer verhaltenen Gesprächsatmosphäre bei Frauen führt. 
$\mathrm{Zu}$ guter Letzt erwies sich der Vergleich der beiden Fälle als herausfordernd. Die Stärke eines Paarvergleichs liegt in der ausgeglichenen Kombination aus deskriptiver Tiefe und analytischer Herausforderung (Tarrow 2010: 246), die weder Einzelfall- noch large-N-Studien bieten können. Der Vergleich kann sich jedoch im Laufe der Forschung teilweise als schwierig erweisen. In den von mir untersuchten Fällen ergaben sich die Herausforderungen des Vergleichs insbesondere aus den regionalen Unterschieden, deren eventuelle Auswirkungen auf die Konflikte und auf meine Forschung ich zu Beginn der Konzeption meiner Untersuchung nicht berücksichtigt habe. Der Norden Ghanas ist sehr viel strukturschwächer als der Süden. Staatliche und formalisierte traditionelle Institutionen, wie die District Assembly oder das CLS, sind in der Northern Region weniger vorhanden als in der Ashanti Region. Die betroffenen Dörfer im BioFuel Africa Fall sind sehr viel verstreuter als im Fall des ScanFarm-Projekts, das formale Bildungssystem ist schwächer und ökonomische Alternativen zur Landwirtschaft sind geringer. Nicht zuletzt aufgrund dieser Faktoren konzentriert sich die wissenschaftliche Forschung in Ghana in den letzten Jahren auf den benachteiligten Norden, was sich in der Haltung der Menschen gegenüber Forscher*innen widerspiegelt: Sie sind "forschungsmüde", wie es eine Kollegin der University of Ghana in Accra ausdrückt. Seit einiger Zeit ist ihre Motivation, bereitwillig Auskunft gegenüber Forscher*innen zu geben, stark gesunken, da sie das Gefühl haben viel zu geben, jedoch nichts zurückzubekommen. Daher war es notwendig, zu Beginn der Befragung der Landnutzer*innen im Dialog mit ihnen herauszufinden, was ihre Erwartungen an meine Forschung sind und transparent zu machen, inwiefern ich diese erfüllen kann oder auch nicht. Des Weiteren könnte der Vergleich der Fälle als schwierig angesehen werden, da eines der beiden Projekte - das BioFuel Africa-Projekt - zum Zeitpunkt der Forschung bereits seit mehreren Jahren beendet war. Dies war jedoch unproblematisch, da ich mich in meiner Untersuchung auf den Zeitraum der Verhandlung der Landvergabe, der Aneignung der Kontrolle über das Land durch den Investor und der Projektimplementierung konzentriere. Unter Berücksichtigung dieser Faktoren, die ein Teil der Forschungsrealität sind, sehe ich die beiden Fälle weiterhin als sehr gut vergleichbar an. 


\subsection{Auswertung und Analyse der Daten}

Mit dem Einverständnis der Interviewtpartner*innen habe ich 40 der insgesamt 47 Interviews aufgezeichnet und anschließend transkribiert. Durch die Aufzeichnung der Interviews konnte ich meinen Gesprächspartner*innen ungeteilte Aufmerksamkeit schenken und gleichzeitig die Fragebögen im Blick behalten. Sieben Interviewpartner*innen entschieden sich gegen eine Aufnahme. $\mathrm{Zu}$ diesen Interviews sowie zu den sieben Gruppendiskussionen habe ich Gesprächsprotokolle angefertigt. Da die meisten dieser Interviews eine Übersetzung aus dem Twi oder Dagbani ins Englische benötigten, hatte ich ausreichend Zeit zur Verfassung der Notizen. Um den Gesprächskontext später gut rekonstruieren zu können, habe ich im Anschluss an jedes Interview zudem eine kurze Beschreibung der Gesprächssituation und -atmosphäre verfasst.

Meine empirischen Daten habe ich mit MaxQDA, einer Software für die computergestützte Analyse qualitativer Daten, ausgewertet. Methodisch orientierte ich mich bei Auswertung der Daten an der qualitativen Inhaltsanalyse (Mayring 2014; Mayring/Fenzl 2014). Die qualitative Inhaltsanalyse ist eine qualitativ-interpretative Methode der Auswertung von Textmaterial, die sich auch zur Erfassung latenter Sinngehalte eignet. Gegenüber anderen Ansätzen der Textanalyse unterscheidet sich die qualitative Inhaltsanalyse dadurch, dass sie entlang klar definierter Kategorien erfolgt. Kategorien können als Kurzformulierungen von Analyseaspekten verstanden werden, deren hierarchische Anordnung in Ober- und Unterkategorien möglich ist (Mayring/Fenzl 2014: 544). Die Kategorien können während der Auswertung induktiv am Material entwickelt sowie vorab deduktiv aus der Theorie hergeleitet werden. Die Zusammenstellung aller für die Analyse relevanten Kategorien im Kategoriensystem, ist der grundlegende Schritt für die Auswertung der Daten. Das Kategoriensystem bildet das zentrale Instrumentarium für die Analyse. Diese berücksichtigt nur Textstellen, denen Kategorien zugeordnet werden können (ebd.). Die Zuordnung der Kategorie zu den entsprechenden Textstellen erfolgt nach zuvor festgelegten Zuordnungsregeln. Der Vorteil dieser Methode der Datenauswertung ist, dass sie streng regelgeleitet verläuft und damit intersubjektiv überprüfbar ist (ebd.: 543).

Für die Entwicklung meines Kategoriensystems habe ich mich am Ablaufmodell induktiver Kategorienbildung und deduktiver Kategorienanwendung von Philipp Mayring und Eva Brunner (2006) orientiert (Abb. 3). Nach diesem Modell folgt als erster Schritt nach der Bestimmung des Ziels der Analyse die 
Festlegung der Analyseeinheiten, die später den Kategorien zugeordnet werden. Als kleinste Einheit legte ich einen Satz fest. Für die Analyse muss dieser immer im Kontext des Absatzes, in den er eingebettet ist, interpretiert werden. Im zweiten Schritt erarbeitete ich mein Kategoriensystem. Dafür leitete ich zunächst deduktiv Kategorien aus dem meiner Studie zugrundeliegenden theoretischen Rahmen ab. Diese Kategorien definierte ich, ordnete ihnen jeweils ein Ankerbeispiel zu und legte eine Kodierregel fest, um sie von anderen Kategorien abzugrenzen. Entlang der deduktiv entwickelten Kategorien begann ich mit der strukturierenden Inhaltsanalyse (Mayring 2002). In dessen Verlauf erweiterte ich die bestehenden Kategorien induktiv um neue Unterkategorien. Die induktiven Kategorien bildete ich aus dem Material heraus, indem ich Themen, Aussagen oder Einstellungen, die wiederholt auftauchten, in Kategorien zusammenfasste. Auch diese neu aus dem Material entwickelten Kategorien definierte ich und ordnete ihnen ein Ankerbeispiel und eine Kodierregel zu. Nachdem ich etwa 30 Prozent des Materials kodiert hatte, überarbeitete ich mein Kategoriensystem noch einmal. Eine weitere Überarbeitung nahm ich nach etwa der Hälfte des Materials vor. Die Validität der Datenkodierung sicherte ich mittels Intrakoderübereinstimmung. Nach der vollständigen Kodierung des Datenmaterials kodierte ich dafür erneut sechs Interviews, die ich nach dem Zufallsprinzip aus jeder Akteursgruppe auswählte, ohne die zuvor kodierten Dokumente eingesehen zu haben. Ich verglich beide Dokumente und glich in der abschließenden Überarbeitung des gesamten Materials bestimmte Zuordnungen, die sich unterschieden oder doppelten, noch einmal an. 


\section{Abbildung 3: Ablaufmodell induktiver Kategorienbildung und deduktiver Kategorien- anwendung}

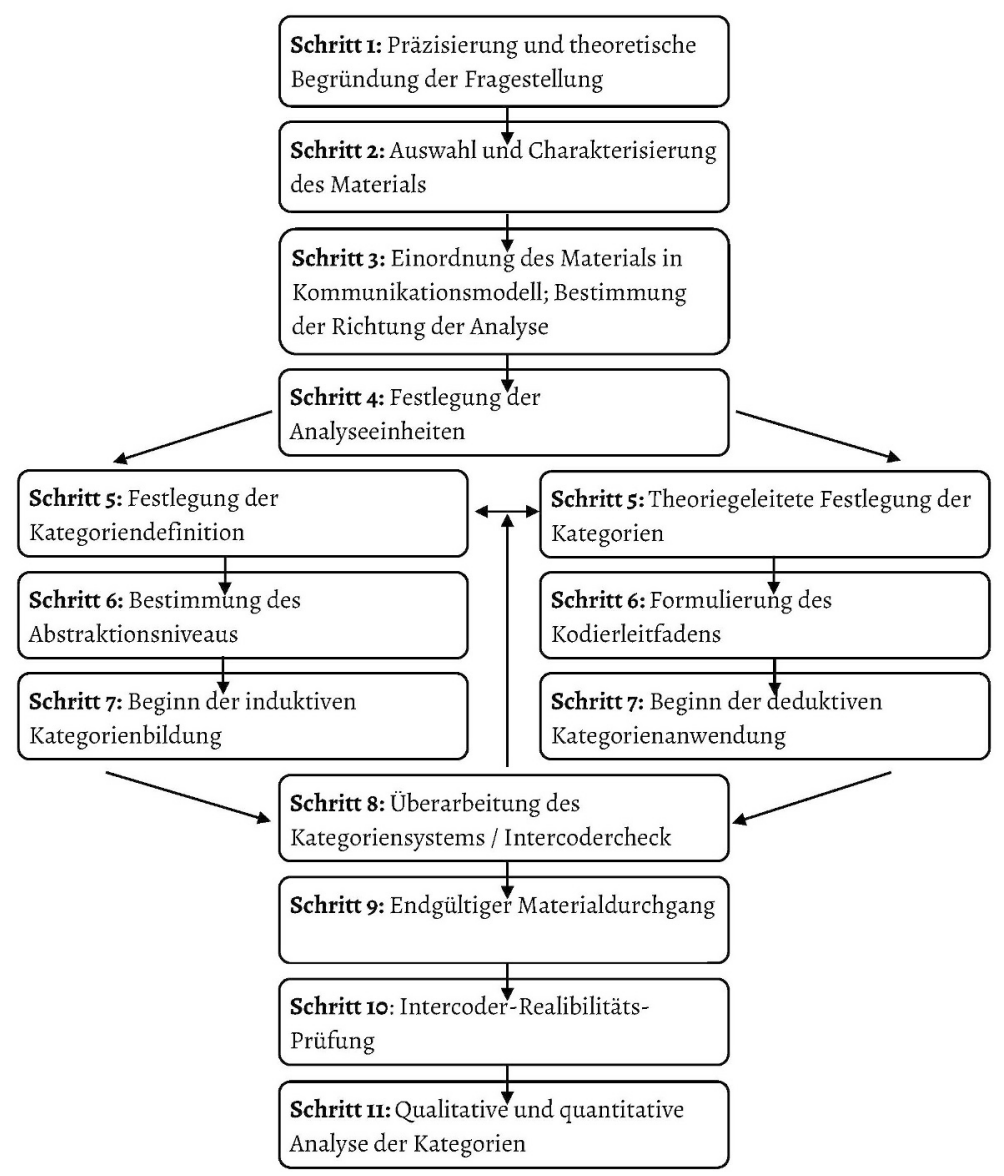

Quelle: Mayring/Brunner 2006 
Folgende Ober- und Unterkategorien leitete ich deduktiv aus der theoretischen Grundlage ab:

\section{Rechtebasierte Zugangsmechanismen}

- Traditionelle Rechte

- Staatliche Rechte

\section{Relationale und strukturelle Zugangsmechanismen}

- Beziehungen

- Netzwerke

- Soziale Zugehörigkeit

\section{Veränderung des Zugangs zu Land im Kontext der Landvergabe}

- Veränderung des rechtebasierten Zugangs

- Veränderung des relationalen und strukturellen Zugangs

\section{Ausübung von Macht und Kontrolle des Zugangs zu Land}

- Sichtbare Macht

- Versteckte Macht

- Unsichtbare Macht

Im Verlauf der induktiven Kategorienentwicklung identifizierte ich keine neuen Oberkategorien, sondern erweiterte die bereits bestehenden deduktiven Kategorien induktiv durch Unterkategorien. Ich differenzierte die rechtebasierten Zugangsmechanismen aus und ergänzte traditionelle Landrechte um die Unterkategorien Gewohnheitsrechte, individuelle Abkommen und formalisierte Landrechte. Staatliche Rechte unterteilte ich in staatlich registrierte traditionelle Landrechte und staatlich registrierte Pachtverträge. Des Weiteren identifizierte ich induktiv Unterkategorien für soziale Zugehörigkeit. Dies waren Geschlecht, Alter, Herkunft und Bildung. Persönliche Beziehungen untergliederte ich in Beziehungen $\mathrm{zu}$ traditionellen Autoritäten, Familienangehörigen und Bekannten. Als Unterkategorien für Netzwerke ergaben sich über die Datenauswertung die Kategorien traditionelle, professionelle und zivilgesellschaftliche Netzwerke. 
Auch für die drei Machtformen entwickelte ich induktiv Unterkategorien. Für die sichtbare Macht waren das Anordnungen gegen den Willen anderer und die Bekräftigung von Entscheidungen gegen Widerstreben. Für die versteckte Macht habe ich die Unterkategorien Ausschluss von Gegner*innen aus Entscheidungsräumen, Bestimmung institutioneller Verfahrensweisen, Beschränkung der Entscheidungsmöglichkeiten von Gegner*innen sowie Kontrolle von Informationen identifiziert. Die unsichtbare Macht habe ich in die Unterkategorien Akzeptanz widersprüchlichen Handels aufgrund gesellschaftlicher Normen, Betonung von Vorteilen trotz verletzter ethischer Interessen und Widersprüche zwischen Handeln und kontrafaktischem Handeln untergliedert.

Die Datenauswertung mit MaxQDA ermöglichte es mir, Zusammenhänge zwischen einzelnen Interviews sowie zwischen Analysekategorien zu erkennen. Die Zusammenhänge zwischen den Interviews waren relevant für den Vergleich der beiden Fälle und erlaubten es, die Unterschiede und Gemeinsamkeiten bezüglich des Handelns traditioneller Autoritäten in Konflikten um Zugang zu Land herauszuarbeiten. Querverbindungen zwischen Analysekategorien lieferten Erkenntnisse bezüglich des Verhältnisses, in dem die unterschiedlichen Zugangsmechanismen zu einander stehen und der Implikationen, die sich daraus für die Kontrolle des Zugangs zu Land ergeben. Ebenso halfen sie dabei, ein Verständnis dafür zu entwickeln, wie sich die unterschiedlichen Machtformen gegenseitig bedingen. Über das Verständnis all dieser Zusammenhänge konnte ich Hypothesen zur Bedeutung des Handelns traditioneller Autoritäten für die Entstehung und den Verlauf von Konflikten um Zugang zu Land bilden.

Die Ergebnisse meiner empirischen Analyse stelle ich in den Kapiteln 7 und 8 dar. Im folgenden Kapitel gebe ich zunächst einen Überblick über den Länderkontext Ghanas mit Schwerpunkt auf Landrechten und der Entwicklung des chieftaincy-Systems. Anschließend daran stelle ich in Kapitel 6 die beiden empirischen Fälle - die Konflikte um ScanFarm und BioFuel Africa vor. 
